\title{
Case Series Review of the Safety and Efficacy of Endoscopic Ultrasound-Guided Splenic Mass Core Biopsy
}

\author{
Sammy Saab ${ }^{1,2}$, Youssef Challita ${ }^{1}$, David Holloman ${ }^{3}$, Kelli Hathaway ${ }^{4}$, Michel Kahaleh ${ }^{5}$ and Jose Nieto ${ }^{3,4}$ \\ Departments of ${ }^{1}$ Surgery, ${ }^{2}$ Medicine, University of California, Los Angeles, CA, ${ }^{3}$ Baptist Medical Center, Jacksonville, FL, ${ }^{4}$ Borland Groover \\ Clinic, Jacksonville, FL, ${ }^{5}$ Weill Cornell Medical College, New York, NY, USA
}

The spleen is a lymphopoietic gland that plays an essential role in the reticuloendothial system. Although uncommon, the spleen can be the site of infectious and malignant diseases, such as lymphoma. ${ }^{1}$ While cross-sectional imaging can help suggest a possible diagnosis, histological and immunochemical evaluation is generally necessary for definitive diagnosis. ${ }^{2}$

Strategies to obtain splenic mass samples include surgery and percutaneous and endoscopic approaches. ${ }^{3.5}$ Percutaneous ultrasound-guided biopsy is the standard technique for intra-abdominal organs; however, in experienced hands, endoscopic ultrasound (EUS)-guided biopsy can also be a safe and effective technique for acquiring tissue samples from intra-abdominal organs. ${ }^{6}$ Utilizing proximity to the gastrointestinal tract, EUS provides a novel approach to sampling a splenic mass as the core biopsy needle traverses less tissue, and may reduce the risk of hemorrhage and hematoma. ${ }^{5}$ Previously, evaluation using EUS-guided biopsy has been limited to cytodiagnosis with fine needle aspiration; however, the use of $19 \mathrm{G}$ core biopsy needles maintains tissue integrity, allowing for immunochemical and histological assessment. ${ }^{7}$

Three patients underwent EUS-guided spleen biopsy (SB) for suspected lymphoma. The median age (range) of the 3 patients was 64 (37-77) years. EUS was performed using a standard linear-array echoendoscope (GF-UE180-AL5; Olympus

Received: October 31, 2017 Revised: November 21, 2017

Accepted: December 11, 2017

Correspondence: Sammy Saab

Departments of Surgery and Medicine, University of California, UCLA Medical Center, 200 Medical Plaza, Suite 214, Los Angeles, CA 90095, USA

Tel: +1-310-206-6705, Fax: +1-310-206-4197, E-mail: SSaab@mednet.ucla.edu ORCID: https://orcid.org/0000-0003-3346-9297.

(cc) This is an Open Access article distributed under the terms of the Creative Commons Attribution Non-Commercial License (http://creativecommons.org/ licenses/by-nc/3.0) which permits unrestricted non-commercial use, distribution, and reproduction in any medium, provided the original work is properly cited.
America, Center Valley, PA, USA). EUS imaging is shown for all 3 patients in the Fig. 1. After identifying the spleen with EUS, a 19 G needle (Acquire ${ }^{\mathrm{TM}} 19 \mathrm{G}$ [Boston Scientific Corporation, Santa Clarita, CA, USA], for case 1, and Sharkcore ${ }^{\mathrm{TM}}$ 19 G [Medtronic, Dublin, Ireland] for cases 2 and 3) was used to obtain core tissue from the splenic mass. One pass with 2 actuations was performed under wet suction. After extraction, core tissue samples were fixed with formalin and sent for flow cytometry. We recorded the total core tissue length, longest intact specimen length, number of cores, and histologic diagnosis.

The median (range) length along the greatest sample dimension was $4.5 \mathrm{~cm}(3.9-7.1 \mathrm{~cm})$. The median (range) number of cores obtained was 8 (8-11). The median (range) total specimen length and longest specimen length was $8.5 \mathrm{~cm}(8-8.5$ $\mathrm{cm})$ and $2.5 \mathrm{~cm}(2-2.5 \mathrm{~cm})$, respectively. Immunochemical staining showed: CD 10+ and BCL-6+; CD 10- and BCL-2+; and $\mathrm{CD} 10+, \mathrm{CD} 20+$, Ki67+, IgH:MYCt (8:14) respectively, in the 3 patients. A definitive diagnosis of lymphoma was made in all patients and there were no complications using EUS-guided SB.

EUS has been described in the assessment of splenic masses but utilization consisted of fine-needle aspiration. ${ }^{5,8}$ Use of core biopsy needles for EUS-guided SB allows for acquisition of larger samples, and enables histological analysis and immunochemical staining. ${ }^{7}$ By using a $19 \mathrm{G}$ rather than a $22 \mathrm{G}$ needle, our novel approach is associated with fewer passes and decreased risk bleeding while maximizing tissue quality. ${ }^{9}$ Using a core needle, biopsies of the spleen have a sensitivity between $86-91 \%$ and a specificity between $91-97 \% .{ }^{4,9}$

In comparing the complications of techniques used for evaluation of splenic masses, surgery is associated with the highest morbidity rates, probably because of infection. ${ }^{10}$ At $8.2 \%$, the 

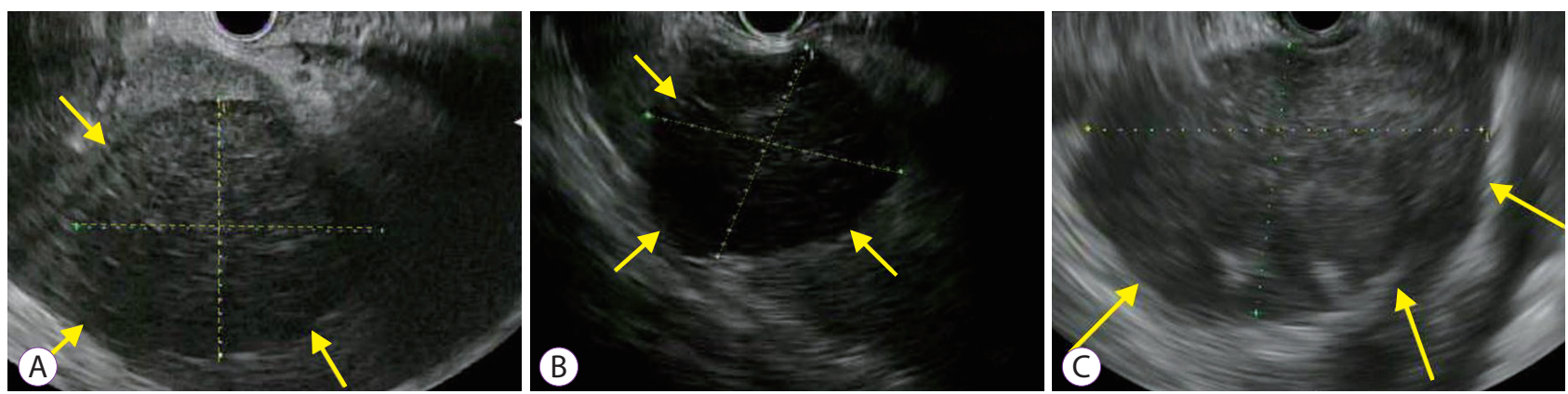

Fig. 1. Endoscopic ultrasound (EUS) findings in patient 1 (A), patient 2 (B), and patient 3 (C). (A) EUS showed a round, homogeneous, hypoechoic splenic mass with smooth borders. The mass measured approximately $45 \times 55 \mathrm{~mm}$. Crosshairs and 3 arrows outline the splenic mass. (B) EUS showed a round, heterogeneous, hypoechoic splenic mass with irregular borders. The mass measured approximately $39 \times 45 \mathrm{~mm}$. The splenic mass is outlined by crosshairs and 3 arrows. (C) EUS showed a round, homogeneous, hypoechoic splenic mass with smooth borders. The mass measured approximately $71 \times 48 \mathrm{~mm}$. The splenic mass is outlined by crosshairs and 3 arrows.

estimated overall complication rate is lower in image-guided percutaneous spleen biopsy. ${ }^{4}$ With use of smaller needles for image-guided percutaneous spleen biopsy, the complication rate decreases to $2.4 \%{ }^{9}$ EUS-guided SB allows for not only direct evaluation of splenic masses, but also surrounding organs. The results of our study showed that EUS-guided SB in experienced hands can be safe and effective in the evaluation of splenic masses.

EUS-guided core biopsy is an effective diagnostic approach in patients with splenic masses. Additional studies are needed to assess the safety of EUS-SB.

\section{Conflicts of Interest}

Michel Kahaleh is a consultant for Boston Scientific, Jose Nieto is a consultant for Boston Scientific and Medtronic. Other authors have no financial conflicts of interest.

\section{Author Contributions}

Conceptualization: Jose Nieto, Sammy Saab

Data curation: JN, Youssef Challita, Kelli Hathaway

Formal analysis: YC, David Holloman, Michel Kahaleh

Investigation: JN, SS, DH, MK

Methodology: JN, DH, MK

Project administration: SS, JN

Resources: JN, DH, MK

Supervision: SS, JN

Validation: SS, JN

Visualization: JN, DH, MK

Writing-original draft: SS, YC, JN

Writing-review \& editing: SS, YC, DH, KH MK, JN

\section{REFERENCES}

1. Wan YL, Cheung YC, Lui KW, Tseng JH, Lee TY. Ultrasonographic findings and differentiation of benign and malignant focal splenic lesions. Postgrad Med J 2000;76:488-493.

2. Sammon J, Twomey M, Crush L, Maher MM, O'Connor OJ. Image-guided percutaneous splenic biopsy and drainage. Semin Intervent Radiol 2012;29:301-310.

3. Tessier DJ, Pierce RA, Brunt LM, et al. Laparoscopic splenectomy for splenic masses. Surg Endosc 2008;22:2062-2066.

4. Olson MC, Atwell TD, Harmsen WS, et al. Safety and accuracy of percutaneous image-guided core biopsy of the spleen. AJR Am J Roentgenol 2016;206:655-659.

5. Fritscher-Ravens A, Mylonaki M, Pantes A, Topalidis T, Thonke F, Swain P. Endoscopic ultrasound-guided biopsy for the diagnosis of focal lesions of the spleen. Am J Gastroenterol 2003;98:1022-1027.

6. Nieto J, Khaleel H, Challita Y, et al. EUS-guided fine-needle core liver biopsy sampling using a novel 19-gauge needle with modified 1-pass, 1 actuation wet suction technique. Gastrointest Endosc 2018;87:469-475.

7. Gimeno-Garcia AZ, Elwassief A, Paquin SC, Sahai AV. Endoscopic ultrasound-guided fine needle aspiration cytology and biopsy in the evaluation of lymphoma. Endosc Ultrasound 2012;1:17-22.

8. Trikudanathan G, Smith S, Mallery JS. Diagnosis of primary splenic flexure schwannoma before surgery based on endoscopic-ultrasound-guided fine-needle biopsy. Clin Gastroenterol Hepatol 2018;16:e29-e30.

9. Liang P, Gao Y, Wang Y, Yu X, Yu D, Dong B. US-guided percutaneous needle biopsy of the spleen using 18-gauge versus 21 -gauge needles. J Clin Ultrasound 2007;35:477-482.

10. Cadili A, de Gara C. Complications of splenectomy. Am J Med 2008;121:371375. 